\title{
Polarisation rotation of slow light with orbital angular momentum in ultracold atomic gases
}

\author{
Julius Ruseckas, ${ }^{1}$ Gediminas Juzeliūnas, ${ }^{1}$ Patrik Öhberg, ${ }^{2}$ and Stephen M. Barnett ${ }^{3}$ \\ ${ }^{1}$ Institute of Theoretical Physics and Astronomy of Vilnius University, A. Goštauto 12, 01108 Vilnius, Lithuania \\ ${ }^{2}$ SUPA, Department of Physics, Heriot-Watt University, Edinburgh EH14 $4 A S$, UK \\ ${ }^{3}$ SUPA, Department of Physics, University of Strathclyde, Glasgow G4 ONG, UK
}

(Dated: June 5, 2007)

\begin{abstract}
We consider the propagation of slow light with an orbital angular momentum (OAM) in a moving atomic medium. We have derived a general equation of motion and applied it in analysing propagation of slow light with an OAM in a rotating medium, such as a vortex lattice. We have shown that the OAM of slow light manifests itself in a rotation of the polarisation plane of linearly polarised light. To extract a pure rotational phase shift, we suggest to measure a difference in the angle of the polarisation plane rotation by two consecutive light beams with opposite OAM. The differential angle $\Delta \alpha_{\ell}$ is proportional to the rotation frequency of the medium $\omega_{\text {rot }}$ and the winding number $\ell$ of light, and is inversely proportional to the group velocity of light. For slow light the angle $\Delta \alpha_{\ell}$ should be large enough to be detectable. The effect can be used as a tool for measuring the rotation frequency $\omega_{\text {rot }}$ of the medium.
\end{abstract}

PACS numbers: 42.50.Gy, 42.50.Fx, 03.75.Kk, 32.70.Jz

\section{INTRODUCTION}

Light can be slowed down by seven orders of magnitude using the Electromagnetically Induced Transparency (EIT) [1, 2, 3]. The EIT makes a resonant, opaque medium transparent by means of quantum interference between the optical transitions induced by the control and probe laser fields. As a result, a weak probe pulse travels slowly and almost without a dissipation in a resonant medium controlled by another laser [1, 2, 3, 4, 5, 6, 7, 8, 9]. Electromagnetically induced transparency was shown not only to slow down dramatically laser pulses [1, 2, 3] but also to store them [10, 11] in atomic gases. Following the proposal of Ref. [12], storage and release of a probe pulse has been demonstrated $[10,11,13]$ by dynamically changing the intensity of the control laser. The possibility to coherently control the propagation of quantum light pulses opens up interesting applications such as generation of nonclassical states in atomic ensembles and reversible quantum memories for light waves [14, 15, 16, 17, 18].

Associated with EIT is a dramatic modification of the reflective properties of the medium. In this paper we analyse the manifestation of the orbital angular momentum (OAM) of light in such a highly dispersive medium. During the last decade optical OAM has received a great deal of interest [19, 20]. However most studies on the topic deal with ordinary (not slow) light. Here we consider the propagation of a slow light with an OAM in a moving atomic medium, the emphasis being placed on a rotating medium, such as a vortex lattice in an atomic Bose-Einstein condensate (BEC). Unlike the previous studies on the slow light in moving media [21, 22, 23, 24, 25, 26, 27], we allow the light to carry an OAM along the propagation direction. We show that the OAM of slow light manifests itself in a rotation of the polarisation plane of a linearly polarised light. To extract a pure rotational phase shift, we suggest to measure a difference in the angles of the rotation of the polarisation plane for two light beams characterised by opposite OAM. The differential angle $\Delta \alpha_{\ell}$ is proportional to the rotation frequency of the medium $\omega_{\text {rot }}$, as well as to the OAM of light. The effect can be used as a tool for measuring the rotation frequency $\omega_{\text {rot }}$ of the medium and/or the OAM of slow light. We should note that rotational phase shifts have been shown to lead to rotation of an image by propagation through a rotating medium (28] and references therein). This suggests that such effects might also be observable in the configuration discussed here.

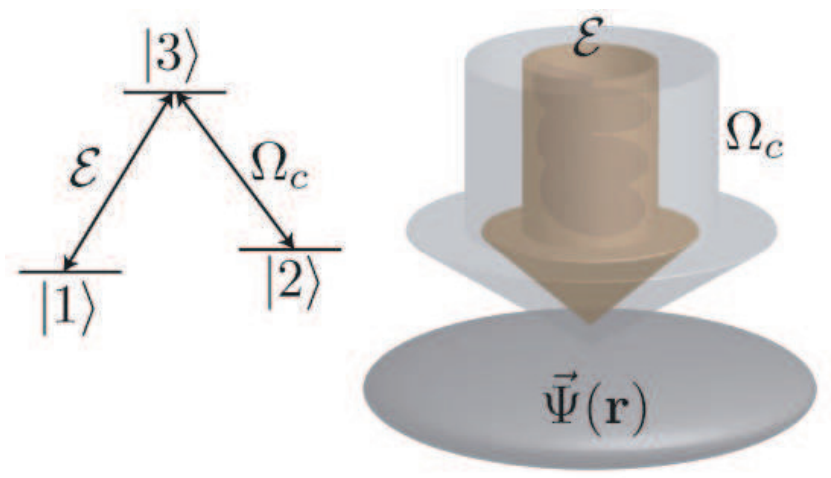

FIG. 1: Slow light propagating in a medium of $\Lambda$-type atoms.

\section{FORMULATION}

Consider an ensemble of $\Lambda$-type atoms characterized by two hyper-fine ground levels 1 and 2 , as well as an electronic excited level 3 (Fig. 1). The translational motion of atoms is described by the three component or vec- 
tor operator for the translational motion, $\vec{\Psi}(\mathbf{r})$, in which the three components, $\Psi_{1} \equiv \Psi_{1}(\mathbf{r}, t), \Psi_{2} \equiv \Psi_{2}(\mathbf{r}, t)$ and $\Psi_{3} \equiv \Psi_{3}(\mathbf{r}, t)$, correspond to the three internal atomic states. The quantum nature of the atoms comprising the medium will, of course, determine whether these field operators obey Bose-Einstein or Fermi-Dirac commutation relations. The atoms interact with two light fields: a strong classical control laser induces a transition $|2\rangle \rightarrow|3\rangle$ and a weaker quantum probe field, which drives a transition $|1\rangle \rightarrow|3\rangle$, see Fig. 1 .

\section{A. Equation for the probe field}

The electric field of the probe beam is

$$
\mathbf{E}(\mathbf{r}, t)=\hat{\mathbf{e}} \sqrt{\frac{\hbar \omega}{2 \varepsilon_{0}}} \mathcal{E}(\mathbf{r}, t) e^{-i \omega t}+\text { H.c. }
$$

where $\omega=c k$ is the central frequency for probe photons, $\mathbf{k}=\hat{\mathbf{z}} k$ is the wave-vector and $\hat{\mathbf{e}} \perp \hat{\mathbf{z}}$ is the unit polarization vector. This field may be considered as either a classical amplitude or a quantum operator. We have chosen the dimensions of the electric field amplitude $\mathcal{E}$ so that its squared modulus represents the number density of probe photons.

The probe field $\mathbf{E}(\mathbf{r}, t)$ obeys the following waveequation

$$
c^{2} \nabla^{2} \mathbf{E}-\frac{\partial^{2} \mathbf{E}}{\partial t^{2}}=\frac{1}{\varepsilon_{0}} \frac{\partial^{2} \mathbf{P}}{\partial t^{2}}
$$

where

$$
\mathbf{P}=\hat{\mathbf{e}} \mu \Psi_{1}^{\dagger} \Psi_{3}+\text { H.c. }
$$

is the polarisation field of atoms, $\mu$ being the dipole moment for the atomic transition $|1\rangle \rightarrow|3\rangle$.

Let us introduce the slowly varying matter fieldoperators:

$$
\begin{aligned}
& \Phi_{1}=\Psi_{1} e^{i \omega_{1} t} \\
& \Phi_{2}=\Psi_{2} e^{i\left(\omega_{1}+\omega-\omega_{c}\right) t} \\
& \Phi_{3}=\Psi_{3} e^{i\left(\omega_{1}+\omega\right) t}
\end{aligned}
$$

where $\hbar \omega_{1}$ is the energy of the atomic ground state 1 and $\omega_{c}$ is the frequency of the control field. The probe field is quasi-monochromatic, so that its amplitude $\mathcal{E} \equiv \mathcal{E}(\mathbf{r}, t)$ varies very slowly in time during an optical cycle. In this case the following equation holds for the slowly-varying amplitude of the probe field:

$$
\left(\frac{\partial}{\partial t}-i \frac{c^{2}}{2 \omega} \nabla^{2}-i \frac{\omega}{2}\right) \mathcal{E}=i g \Phi_{1}^{\dagger} \Phi_{3},
$$

where the parameter

$$
g=\mu \sqrt{\omega / 2 \varepsilon_{0} \hbar}
$$

characterizes the strength of coupling of the probe field with the atoms. Note that, unlike in the usual treatment of slow light [14, 15, 16, 17, 24, 25, 26, 27], we have retained the second-order derivative $\nabla^{2}$ in the equation of motion (7). This allows us to account for the fast changes of $\mathcal{E}$ in a direction perpendicular to the wave-vector $\mathbf{k}$, i.e. in the $x y$-plane. Therefore, our analysis can be applied to the twisted beams of light $\mathcal{E}(\mathbf{r}, t) \sim \exp (i \ell \varphi)$ carrying an OAM of $\hbar \ell$ per photon.

\section{B. Equations for the matter field-operators}

In the following we shall adapt a semiclassical picture in which both electromagnetic and matter field operators are replaced by c-numbers. The equations for the matter fields are then:

$$
\begin{aligned}
\hat{K} \Phi_{1} & =V_{1}(\mathbf{r}) \Phi_{1}-\hbar g \mathcal{E}^{*} \Phi_{3} \\
\hat{K} \Phi_{3} & =\hbar \omega_{31} \Phi_{3}+V_{3}(\mathbf{r}) \Phi_{3}-\hbar \Omega_{c} \Phi_{2}-\hbar g \mathcal{E} \Phi_{1} \\
\hat{K} \Phi_{2} & =\hbar \omega_{21} \Phi_{2}+V_{2}(\mathbf{r}) \Phi_{2}-\hbar \Omega_{c}^{*} \Phi_{3}
\end{aligned}
$$

with

$$
\hat{K}=i \hbar \frac{\partial}{\partial t}+\frac{\hbar^{2}}{2 m} \nabla^{2}
$$

Here $m$ is the atomic mass, $V_{j}(\mathbf{r})$ is the trapping potential for an atom in the electronic state $j(j=1,2,3), \Omega_{c}$ is the Rabi-frequency of the control laser driving the transition $|2\rangle \rightarrow|3\rangle ; \omega_{21}=\omega_{2}-\omega_{1}+\omega_{c}-\omega$ and $\omega_{31}=\omega_{3}-\omega_{1}-\omega$ are, respectively, the frequencies of electronic detuning from the two- and one-photon resonances. In the limit of infinite mass we can ignore the spatial derivatives in these equations which then reduce to the familiar equations for the probability amplitudes describing the coherent excitation of three-level atoms in the $\Lambda$ configuration [8]. For us, however, retention of this center of mass motion is essential in order to describe light dragging effects. Note also that the equation of motion (9) for $\Phi_{1}$ does not explicitly accommodate collisions between the groundstate atoms. In the case of a BEC where the state 1 is mostly populated, the collisional effects can be included replacing $V_{1}(\mathbf{r})$ by $V_{1}(\mathbf{r})+g_{11}\left|\Phi_{1}\right|^{2}$ in Eq. (9) to yield a mean-field equation for the condensate wave-function $\Phi_{1}$, where $g_{11}=4 \pi \hbar^{2} a_{11} / m$ and $a_{11}$ is the scattering length between the condensate atoms in state 1. In Eqs. (9)-(11) the coupling with the probe and control fields has been written using the rotating wave approximation. Therefore the last term in Eq. (9) has a positive frequency part of the probe field (i.e. $\mathcal{E}^{*}$ ), whereas the last term in Eq. (10) has a negative frequency part $(\mathcal{E})$. For the same reason, Eq. (10) contains a factor $\Omega_{c}$, whereas Eq. (11) contains a factor $\Omega_{c}^{*}$.

Equation (11) relates $\Phi_{3}$ to $\Phi_{2}$ as:

$$
\Phi_{3}=\frac{1}{\hbar \Omega_{c}^{*}}\left(-\hat{K}+V_{2}(\mathbf{r})+\hbar \omega_{21}\right) \Phi_{2} .
$$


Initially the atoms are in the ground level 1 and the Rabi frequency of the probe field is considered to be much smaller than $\Omega_{c}$. Consequently one can neglect the last term in Eq. (9) that causes depletion of the ground level 1, giving $\hat{K} \Phi_{1}=V_{1}(\mathbf{r}) \Phi_{1}$. In the case of a BEC the wave-function $\Phi_{1}=\sqrt{n} \exp \left(i S_{1}\right)$ represents an incident variable determining the atomic density $n$ and velocity field $\hbar \nabla S_{1} / m$.

\section{PROPAGATION OF PROBE BEAM}

\section{A. Adiabatic approximation}

Suppose the control and probe beams are close to the two-photon resonance. Application of the two beams cause electromagnetically induced transparency (EIT) [1, 2, [3, 4, 5, 6, 6, 8, 9] in which the transitions $|2\rangle \rightarrow|3\rangle$ and $|1\rangle \rightarrow|3\rangle$ interfere destructively preventing population of the excited state 3 . The adiabatic approximation is obtained neglecting the excited state population in Eq. (10) to yield:

$$
\Phi_{2}=-g \frac{\Phi_{1}}{\Omega_{c}} \mathcal{E}
$$

Equations (7), (13) and (14) provide a closed set of the equations for the electric field amplitude $\mathcal{E}$ :

$$
\begin{aligned}
& \left(\frac{\partial}{\partial t}-i \frac{c^{2}}{2 \omega} \nabla^{2}-i \frac{\omega}{2}\right) \mathcal{E}= \\
& -i \frac{g^{2} \Phi_{1}^{*}}{\hbar \Omega_{c}^{*}}\left(-\hat{K}+V_{2}(\mathbf{r})+\hbar \omega_{21}\right) \frac{\Phi_{1} \mathcal{E}}{\Omega_{c}} .
\end{aligned}
$$

This equation suffices to describe a wide variety of phenomena. In particular it can be used to model light storage by introducing time-dependence in $\left|\Omega_{c}\right|[12]$ and light dragging due to spatial variation of $\Omega_{c}$ and/or $\Phi_{1}$. Here we shall be especially interested in image rotation associated with spatial variations of $\Phi_{1}$, corresponding to a moving medium and with light beams carrying orbital angular momentum so that $\mathcal{E} \propto e^{i \ell \phi}$.

\section{B. Co-propagating control and probe beams}

Suppose the probe and control beams co-propagate:

$$
\begin{aligned}
\mathcal{E}(\mathbf{r}, t) & =\tilde{\mathcal{E}}(\mathbf{r}, t) e^{i k z} \\
\Omega_{c}(\mathbf{r}, t) & =\Omega(\mathbf{r}, t) e^{i k_{c} z}
\end{aligned}
$$

where $k_{c}=\omega_{c} / c$ is the wave-number of the control beam. For paraxial beams the amplitudes $\tilde{\mathcal{E}}(\mathbf{r}, t)$ and $\Omega(\mathbf{r}, t)$ depend only weakly on the propagation direction $z$. Furthermore the amplitude of the control field $\Omega(\mathbf{r}, t)$ is considered to change weakly in the transverse direction as well. Neglecting the spatial dependence of $\Omega(\mathbf{r}, t)$, the equation (15) for the probe field takes the form

$\frac{\partial}{\partial t} \tilde{\mathcal{E}}+v_{g}\left[\frac{\partial}{\partial z}+\left(\frac{1}{v_{g}}-\frac{1}{c}\right)\left(\mathbf{v}_{a} \cdot \nabla+i \delta\right)-i \frac{1}{2 k} \nabla_{\perp}^{2}\right] \tilde{\mathcal{E}}=0$

where we have replaced $\nabla^{2}$ by its transverse part $\nabla_{\perp}^{2}=$ $\partial^{2} / \partial x^{2}+\partial^{2} / \partial y^{2}$ because of the paraxial approximation. Here

$$
v_{g}=\frac{c\left|\Omega_{c}(\mathbf{r}, t)\right|^{2}}{\left|\Omega_{c}(\mathbf{r}, t)\right|^{2}+g^{2} n(\mathbf{r})}
$$

is the radiative group velocity,

$$
\mathbf{v}_{a}=-\frac{i \hbar}{m} \frac{1}{\Phi_{1}} \nabla \Phi_{1}=\frac{\hbar}{m} \nabla S_{1}-\frac{i \hbar}{2 m} \frac{1}{n} \nabla n
$$

is a complex atomic velocity field, and

$$
\delta=\omega_{21}+\frac{1}{\hbar}\left(V_{2}(\mathbf{r})-V_{1}(\mathbf{r})\right)
$$

is the two-photon frequency mismatch. As the beams co-propagate, the ordinary two-photon Doppler shift $\mathbf{v}_{a}$. $\hat{\mathbf{z}}\left(k-k_{c}\right)$ and two photon recoil $\hbar\left(k-k_{c}\right)^{2} / 2 m$ are of little importance and hence have been omitted. Furthermore, the radiative velocity $v_{g}$ is much larger than the velocity of the atomic recoil $v_{\text {rec }} \equiv \hbar k / m$, so the latter $v_{\text {rec }}$ has been neglected in the equation of motion (18).

The term with the spatial derivative $\partial / \partial z$ in Eq. (18) describes the radiative propagation along the $z$-axis with the group velocity $v_{g}$. On the other hand, the term containing $\mathbf{v}_{a} \cdot \nabla$ represents the dragging of light by a moving medium. The amount of such a dragging is determined by the difference in the group velocity and the speed of light. For fast light there is no dragging, but for slow light a substantial dragging is possible.

\section{Rotating medium}

Consider a probe beam carrying an orbital angular moment $(\mathrm{OAM}) \hbar \ell$ per photon along the propagation axis $\tilde{\mathcal{E}}(\mathbf{r}, t) \sim e^{i \ell \varphi}$, where $\varphi$ is the azimuthal angle. Suppose the medium is rotating as a rigid body with a frequency $\omega_{\text {rot }}$ in the $x y$ plane. For instance, an atomic vortex lattice exhibits such rigid body rotation on a coarse grained scale [29]. In such a situation the linear velocity is $\mathbf{v}_{a}=\omega_{\operatorname{rot}} \rho \mathbf{e}_{\varphi}$, where $\mathbf{e}_{\varphi}$ is the unit vector along the azimuthal direction and $\rho=\sqrt{x^{2}+y^{2}}$ is the cylindrical radius. Under these conditions the dragging term featured in the equation of motion (18) takes the form

$$
\mathbf{v}_{a} \cdot \nabla \tilde{\mathcal{E}}(\mathbf{r}, t)=i l \omega_{\operatorname{rot}} \tilde{\mathcal{E}}(\mathbf{r}, t) .
$$

Suppose the atomic density (and hence the group velocity) is approximately constant in the transverse direction [30], so that the group velocity depends on the propagation coordinate only: $v_{g}=v_{g}(z)$. Using Eqs. (18) and 
(22), the total phase shift then acquired by the beam propagating along the $z$-axis is

$$
\theta_{\ell}=\left(\ell \omega_{\mathrm{rot}}+\delta\right) \int_{z_{0}}^{z}\left(\frac{1}{v_{g}\left(z^{\prime}\right)}-\frac{1}{c}\right) d z^{\prime}
$$

where $z_{0}$ is the entrance point into the medium. The first term in $\left(\ell \omega_{\text {rot }}+\delta\right)$ represents the rotational frequency shift due to the orbital angular momenta $\hbar \ell$ of the probe photons. If the control and probe fields are circularly polarised, the second term accommodates the rotational two-photon frequency shift $\pm 2 \omega_{\text {rot }}$, as well as the residual detuning $\delta^{\prime}$

$$
\delta= \pm 2 \omega_{\text {rot }}+\delta^{\prime}
$$

where the sign of $\pm 2 \omega_{\text {rot }}$ depends on the polarisations of the control and probe field. The factor 2 appears because the control and probe beams are considered to have opposite circular polarisations leading a double frequency shift. In the slow-light experiments involving a $\Lambda$ scheme [10, 11] it is quite common for the control and probe fields to have the opposite circular polarisations which drive the atomic transitions with $\Delta m= \pm 1$. The residual detuning $\delta^{\prime}$ depends neither on the orbital angular momentum of a photon nor on its polarisation and is hence of little importance for the subsequent discussion.

\section{Polarisation rotation of slow light with OAM}

A characteristic feature of an EIT medium is that only one circularly polarised component of light (say left polarised light) is slowed down. The right circularly polarised light has a forbidden resonant transition to the excited state and is propagating with a group velocity close to the speed of light in vacuum. If the medium rotates and the probe beam carries an OAM, the left circularly polarised beam of light acquires a large rotational frequency shift because of its small group velocity. On the other hand, the right polarised light is a fast light and hence experiences almost no rotational frequency shift. If the incoming light beam is linearly polarised, this leads to the rotation of the polarisation plane by an angle $\alpha_{\ell}=\left(\theta_{\ell}+\theta_{\text {fast }}\right) / 2$, where $\theta_{\text {fast }}$ is the dynamical phase shift acquired by the fast (right polarised) light, $\theta_{\ell}$ being the phase shift acquired by the slow (left polarised) light given by Eq. (23). To extract a pure rotational phase shift, we suggest measuring the difference in the angles of the polarisation plane rotation for two consecutive probe beams characterised by opposite OAM

$$
\Delta \alpha_{\ell}=\alpha_{\ell}-\alpha_{-\ell}=\ell \omega_{\operatorname{rot}} \int_{z_{0}}^{z}\left(\frac{1}{v_{g}\left(z^{\prime}\right)}-\frac{1}{c}\right) d z^{\prime}
$$

where we have taken advantage of the fact that $\theta_{\text {fast }}$ and $\delta$ do not depend on the winding number $\ell$.

The angle $\Delta \alpha_{l}$ is proportional to the rotation frequency of the medium $\omega_{\text {rot }}$ and to the winding number $\ell$. Therefore the effect is due to the orbital angular momentum of slow light. Furthermore, the angle $\Delta \alpha_{\ell}$ is inversely proportional to the group velocity of light. This suggests that for slow light the angle $\Delta \alpha_{l}$ should be large enough to be detectable. Let us make some numerical estimates for typical BEC / slow light experimental parameters. In the experiment by Hau et al [1] the radiative group velocity is $v_{g}=17 \mathrm{~m} / \mathrm{s}$, whereas the length of a cigar shaped BEC is $\Delta z=z_{1}-z_{0}=0.3 \mathrm{~mm}=3 \times 10^{-4} \mathrm{~m}$. Typically the transverse frequency of an elongated trap is $\omega \sim 2 \pi \times 10^{2}$, so the rotation frequency $\omega_{\text {rot }}$ should be of the same order. Thus the difference in the angle of the polarisation plane rotation is $\Delta \alpha_{\ell} \approx 4 \pi \ell \times 10^{-5}$. This is a small angle, yet by no means beyond current experimental expertise [31]. For fast light the corresponding angle $\Delta \alpha_{\ell}$ should be far too small to be observed.

\section{CONCLUSIONS}

In the present paper we have considered the propagation of a slow light with an orbital angular momentum in a moving atomic medium. We have derived a general equation of motion and applied it to propagation of a slow light with an orbital angular momentum in a rotating medium. We have shown that the orbital angular momentum of slow light manifest itself in a rotation of the polarisation plane of a linear polarised light. To extract a pure rotational phase shift, we suggest to measure the difference in the angles of the polarisation plane rotation for two consecutive beams of probe light characterised by opposite OAM. The differential angle $\Delta \alpha_{\ell}$ is proportional to the rotation frequency of the medium $\omega_{\text {rot }}$, as well as to the winding number $\ell$. The effect can be used as a tool for measuring the rotation frequency $\omega_{\text {rot }}$ of the medium and/or the orbital angular momentum of slow light.

\section{Acknowledgments}

This work was supported by the Royal Society, the UK Engineering and Physical Sciences Research Council, the Royal Society of Edinburgh and by the Wolfson Foundation.
[1] L. V. Hau, S. E. Harris, Z. Dutton, and C. H. Behroozi, Nature (London) 397, 594 (1999).
[2] M. M. Kash, V. A. Sautenkov, A. S. Zibrov, L. Hollberg, 
G. R. Welch, M. D. Lukin, Y. Rostovtsevand, E. S. Fry, and M. O. Scully, Phys. Rev. Lett. 82 (1999).

[3] D. Budker, D. F. Kimball, S. M. Rochester, and V. V. Yashchuk, Phys. Rev. Lett. 83, 1767 (1999).

[4] S. E. Harris, J. E. Field, and A. Imamoğlu, Phys. Rev. Lett. 64, 1107 (1990).

[5] K. J. Boller, A. Imamoğlu, and S. E. Harris, Phys. Rev. Lett. 66, 2593 (1991).

[6] E. Arimondo, Progress in Optics (Elsevier, Amsterdam, 1996), p. 257.

[7] S. E. Harris, Phys. Today 50, 36 (1997).

[8] M. O. Scully and M. S. Zubairy, Quantum Optics (Cambridge University Press, Cambridge, 1997).

[9] K. Bergmann, T. Theuer, and B. W. Shore, Rev. Mod. Phys. 70, 1003 (1998).

[10] C. Liu, Z. Dutton, C. H. Berhoozi, and L. V. Hau, Nature (London) 409, 490 (2001).

[11] D. F. Phillips, A. Fleischhauer, A. Mair, R. L. Walsworth, and M. D. Lukin, Phys. Rev. Lett. 86, 783 (2001).

[12] M. Fleischhauer and M. D. Lukin, Phys. Rev. Lett. 84, 5094 (2000).

[13] A. V. Turukhin, V. S. Sudarshanam, M. S. Shahriar, J. A. Musser, B. S. Ham, and P. R. Hemmer, Phys. Rev. Lett. 88, 023602 (2001).

[14] M. D. Lukin, S. F. Yelin, and M. Fleischhauer, Phys. Rev. Lett. 84, 4232 (2000).

[15] G. Juzeliūnas and H. J. Carmichael, Phys. Rev. A 65, 021061(R) (2002).

[16] M. D. Lukin, Rev. Mod. Phys. 75, 457 (2003).

[17] M. Fleischhauer, A. Imamoglu, and J. P. Marangos, Rev. Mod. Phys. 77, 633 (2005).
[18] N. S. Ginsberg, S. R. Garner, and L. V. Hau, Nature (London) 445, 623 (2007).

[19] L. Allen, M. Padgett, and M. Babiker, Prog. Opt. 39, 291 (1999).

[20] L. Allen, S. M. Barnett, and M. J. Padgett, Optical Angular Momentum (Institute of Physics, Bristol, 2003).

[21] U. Leonhardt and P. Piwnicki, Phys. Rev. Lett. 84, 822 (2000).

[22] J. Fiurasek, U. Leonhardt, and R. Parentani, Phys. Rev. A 65, 011802 (2002).

[23] P. Öhberg, Phys. Rev. A 66, 021603(R) (2002).

[24] M. Fleischhauer and S. Gong, Phys. Rev. Lett. 88, 070404 (2002).

[25] G. Juzeliūnas, M. Mašalas, and M. Fleischhauer, Phys. Rev. A 67, 023809 (2003).

[26] I. Carusotto, M. Artoni, G. C. L. Rocca, and F. Bassani, Phys. Rev. A 68, 063819 (2003).

[27] F. Zimmer and M. Fleischhauer, Phys. Rev. Lett. 92, 253201 (2004).

[28] M. Padgett, G. Whyte, J. Girkin, A. Wright, L. Allen, P. Öhberg, and S. M. Barnett, Opt. Lett. 31, 2205 (2006).

[29] L. Pitaevskii and S. Stringari, Bose-Einstein Condensation (Clarendon Press, Oxford, 2003).

[30] In many BEC experiments the external trap is harmonic. With increasing rotation the trapped atomic cloud acquires a pancake shape (with holes if the vortex lattice is formed). Therefore the density is on average constant over a reasonably large area in the central region of the trap.

[31] M. Lintz, J. Guena, and M. Bouchiat, arXiv:physics/0610035 (2006). 